
\title{
$\begin{array}{ll}\text { Research Square } & \begin{array}{l}\text { Preprints are preliminary reports that have not undergone peer review. } \\ \text { They should not be considered conclusive, used to inform clinical practice, } \\ \text { or referenced by the media as validated information. }\end{array}\end{array}$
}

\section{Urothelial Carcinoma of the Graft Kidney With Molecular Analyses: A Rare Case Report}

\author{
Joyce Chen \\ Icahn School of Medicine at Mount Sinai \\ Kenneth Haines \\ Icahn School of Medicine at Mount Sinai \\ William Lam \\ Icahn School of Medicine at Mount Sinai \\ Asha Reddy \\ Icahn School of Medicine at Mount Sinai \\ Meenakshi Mehrotra \\ Icahn School of Medicine at Mount Sinai \\ Jane Houldsworth \\ Icahn School of Medicine at Mount Sinai \\ Qiusheng Si ( $\square$ qiusheng.si@mountsinai.org ) \\ Icahn School of Medicine at Mount Sinai
}

\section{Case Report}

Keywords: High grade papillary urothelial carcinoma, transplant kidney, microsatellite instability, CDKN2A/CDKN2B

Posted Date: March 22nd, 2021

DOI: https://doi.org/10.21203/rs.3.rs-318637/v1

License: (c) (i) This work is licensed under a Creative Commons Attribution 4.0 International License. Read Full License 


\section{Abstract}

\section{BACKGROUND}

Malignancy after transplantation is a leading cause of death in kidney transplant recipients. However, donor-derived malignancies are rarely reported. We herein report a high grade papillary urothelial carcinoma of the graft kidney, involving the graft ureter and native urinary bladder, invading one left obturator lymph node, and sparing the two native kidneys and ureters.

\section{CASE PRESENTATION}

62-year-old female with history of kidney transplantation more than 30 years ago, who presented with urinary tract infection, acute renal failure, and hydronephrosis of the transplant kidney. Anterograde nephrostogram showed a large filling defect in the lower pole of the transplant kidney and a filling defect in the proximal 3-4 cm of ureter. Biopsy taken from the renal pelvic mass showed a high grade urothelial carcinoma. She underwent hysterectomy, bilateral salpingo-oophorectomy, nephrectomy of native kidneys and transplant kidney, cystectomy and bilateral pelvic lymph node dissections. Histology showed a high grade papillary urothelial carcinoma of the graft kidney, involving the graft ureter, native urinary bladder and one left obturator lymph node and sparing the two native kidneys and ureters. Short tandem repeat (STR) analysis confirmed the tumor was of donor origin. The next-generation sequencing identified amplification of TERT and loss of CDKN2A/CDKN2B in the primary tumor.

\section{CONCLUSION}

While it is known that transplant recipients have an increased risk of urothelial carcinoma compared to the general population, the lack of the well-documented risk factors, such as older age at transplantation, BK polyomavirus infection, and prolonged post-transplantation history and dissemination of the tumor in this case shed light on the de novo tumorigenesis of the graft kidney within the host microenvironment. Amplification of Telomerase reverse transcriptase (TERT) and loss of cyclin dependent kinase inhibitor 2A/2B (CDKN2A/CDKN2B) detected in the tumor by next gene sequencing suggests that they may play an important role in this case.

\section{Introduction}

Kidney transplantation has been established as the treatment of choice for patients with end-stage renal disease ${ }^{1,2}$. With the advance of immunosuppressive regimens, transplant patients have a better quality of life and significant survival benefit compared to those continuing on dialysis. However, transplant patients have a higher risk of developing secondary malignancies post transplantation, owing to the extended life expectancy and chronic immunosuppressive status ${ }^{3}$. Malignancy after transplantation has become the third leading cause of death in renal transplant recipients ${ }^{4,5}$. Compared to the normal population, post-transplant patients have a 7-fold increased risk of developing renal cell carcinoma (RCC) in the native kidney and a significantly worse prognosis. Risk factors include end-stage renal disease, 
longer time on dialysis, and older ages at transplant. These patients also have a 3-fold increased risk of developing urothelial carcinoma (UC) in the native bladder, which is associated with BK polyomavirus. However, donor-derived UC is rarely reported ${ }^{6,7}$. We herein report a high grade papillary urothelial carcinoma of the graft kidney, involving the graft ureter and native urinary bladder, invading one left obturator lymph node, and sparing the two native kidneys and ureters.

\section{Case Report}

A 62-year-old female, who underwent living-related donor renal transplant in 1983. No rejection was seen post-transplant, and the transplant kidney functioned well. In 2017, she presented with urinary tract infection, and further examination revealed hydronephrosis and acute renal failure. She was treated with Cefapime for a week and bladder cytology reported positive for malignant cells. Anterograde nephrostogram showed a large filling defect in the lower pole of the transplant kidney and a filling defect in the proximal 3-4 cm of ureter. One week later, she underwent an ureteroscopy, and multiple biopsies were taken from the renal pelvic mass. Pathology reported high grade urothelial carcinoma. Later on, the patient was found to have extensive bladder tumors; she underwent hysterectomy, bilateral salpingooophorectomy, nephrectomy of native kidneys and transplant kidney, cystectomy and bilateral pelvic lymph node dissections. Post operation follow-up, pelvic magnetic resonance imaging demonstrated a large liver mass which was confirmed by biopsy to be metastatic urothelial carcinoma. Immunotherapy was initiated and the liver metastasis responded well to the immunotherapy with dramatic reduction in size of liver mass. So far, the patient lives well with dialysis.

The Fig. 1A showed left native kidney, two native ureters, and one donor kidney connected to the native urinary bladder via a donor ureter. Upon sectioning, a $4.5 \times 2.5 \times 1.5 \mathrm{~cm}$ well-circumscribed flesh-colored mass was found in the renal pelvis of the graft kidney, a $1.2 \times 1.4 \times 2.5 \mathrm{~cm}$ flesh-colored mass found encompassing the entire lumen of the proximal donor ureter, and over fifty tan small discrete polypoid nodules, up to $1.5 \mathrm{~cm}$ in size, were found carpeting the wall of native bladder (Fig. 1B and 1C). Bilateral native kidneys were small in size $(5.0 \times 3.5 \times 1.5 \mathrm{~cm}$ and $6.0 \times 4.0 \times 2.0 \mathrm{~cm}$, respectively), and showed a thinned cortex, a poorly delineated corticomedullary junction, and a renal pelvis largely replaced by fatty tissue. No mass was identified in native kidneys or ureters. Histology demonstrated a high grade papillary UC involving the graft kidney, graft ureter and the native bladder, with metastasis to one of thirteen obturator lymph nodes, sparing two native kidneys and ureters (Fig. 2). The high grade papillary UC was mostly confined in the pelvicalyceal system only with focal invasion into subepithelial connective tissue. The non-invasive ureteral tumor highly resembled the morphological features of UC in the renal pelvis. In the bladder, multifocal small polypoid lesions showed exophytic papillary UC, The native kidney showed thyroidization with tubular casts, calcium oxalate deposition, global and segmental glomerular sclerosis, and arteriosclerosis. Diffuse interstitial fibrosis, tubular atrophy, and dense outer cortical mononuclear inflammationwere also present (not shown).

Since microsatellite instability (MSI) is commonly seen in upper urinary tract $\mathrm{UC}^{8}$, we performed immunohistochemistry and found that MLH1, MLH2, PMS2 and MSH6 expression were retained in the 
UC of renal pelvis, donor ureter, and urinary bladder (not shown). BK polyomavirus has been shown as risk factors in post-transplant UC. However, the donor and recipient kidneys were negative for BK viral infection by SV4O IHC (Fig. 3). In order to determine the origin of the UC, STR analysis was performed on extracted DNA from the uninvolved native uterus as the control for recipient and representative apparent normal donor kidney as the control for donor. Of the 15 autosomal STRs, nine were informative. Review of these informative loci in the DNA extracted from the donor kidney tumor, ureter tumor, and bladder tumor indicated that $83 \%, 84 \%$, and $80 \%$,respectively, were of donor origin. These data would suggest that the tumor had drop metastasized to the bladder through the donor ureter. DNA extracted from the lymph node with evidence of metastasis showed only $8 \%$ donor origin, possibly suggestive of low tumor cellularity in the lymph node sampled.

DNA extracted from the donor kidney tumor and the lymph node with evidence of metastasis were submitted to mutation profiling of 147 genes, and the results are provided in Table 1 . The donor kidney tumor showed amplification of TERT and loss of CDKN2A/CDKN2B, both of which are genomic alterations found in approximately $7 \%$ and $25 \%$ of bladder cancers respectively (https://www.cbioportal.org). This specimen also showed a missense variant (p.N596S) in MSH2 at a low VAF (3.5\%). This same variant was detected in the lymph node that had evidence of metastasis but at a VAF of $37 \%$. It also showed two other missense variants in MSH6 and FANCA with VAFs of $34 \%$ and $41 \%$ respectively. Interpretation of these variants would suggest these are of uncertain significance (VUS), potentially of germline origin. Given the low donor contribution in this lymph node specimen from the STR analyses (8\%), it is further suggested that these are of recipient (native) germline origin. The low VAF MSH2 detected in the donor kidney tumor confirms the $7 \%$ recipient (native) contribution detected in this specimen. Thus, for the lymph node, no apparent somatic alterations were detected, possibly due to the overall low tumor cellularity of this specimen.

Table 1

Mutation Profiling by Next Generation Sequencing (NGS)

\begin{tabular}{|c|c|c|c|c|c|}
\hline Specimen & Gene & Alteration & VAF & Coverage & Significance \\
\hline \multicolumn{6}{|c|}{ Donor kidneytumor (50\% estimated tumor cellularity, $83 \%$ donor) } \\
\hline & $\mathrm{MSH} 2$ & c. $1787 A>G ; p . N 596 S$ & $3.5 \%$ & $1576 x$ & Uncertain (germline) \\
\hline & TERT & Amplification (18.8x) & & & Gain-of-function \\
\hline & CDKN2A & Loss $(0.25 x)$ & & & Loss-of-function \\
\hline & CDKN2B & Loss $(0.23 x)$ & & & Loss-of-function \\
\hline \multicolumn{6}{|c|}{ Recipient lymph node with metastasis ( $50 \%$ estimated tumor cellularity, $8 \%$ donor) } \\
\hline & MSH2 & c. $1787 A>G ; p . N 596 S$ & $37 \%$ & $1552 x$ & Uncertain (germline) \\
\hline & MSH6 & c. $116 \mathrm{G}>\mathrm{A} ;$ p.G39E & $34 \%$ & $193 x$ & Uncertain (germline) \\
\hline & FANCA & c. $1772 \mathrm{G}>\mathrm{A} ; \mathrm{p} . \mathrm{R} 591 \mathrm{Q}$ & $41 \%$ & $3055 x$ & Uncertain (germline) \\
\hline
\end{tabular}




\section{Discussion}

Malignancy after transplantation is a leading cause of death in renal transplant recipients. Posttransplant malignancies develop within three to five years post transplantation and the mean age of disease onset is 40-years. Following renal transplant, kidney cancer almost exclusively occurs in native kidneys with papillary RCC as the most common type ${ }^{9}$. However, donor-derived malignancies are rarely reported 6,7 . Our case is a rare donor-derived UC in the graft kidney with a few very interesting facts. Firstly, the patient received transplant in her 20s and developed a donor-derived UC 34 years later. Secondly, she lacked the well-documented risk factors of secondary malignancy post-transplant, such as older age at transplantation and BK virus infection ${ }^{10-13}$. Thirdly, her two native kidneys with end-stage renal disease were spared from the tumorigenesis, although they bear a 100-fold and 4.4-fold increased risk of developing RCC and UC compared to the general population ${ }^{3}$.

There are three possibilities regarding the UC origin. One possibility is that the tumor originated in the native bladder, the most common site of post-transplant UC, and spread anterograde to the donor ureter and graft kidney. Another possibility is that the primary tumor originated from the renal pelvis, given the greatest size of the renal pelvic tumor, and drop metastasized to the donor ureter and native bladder. The last possibility is concurrent UC of the renal pelvis and urinary bladder. The supporting evidence for the last possibility stems from the fact that the tumors from those two sites are quite different in terms of size and invasiveness. To answer these questions, we performed STR analysis, a molecular analysis of 16-STR widely used in medicine for establishing paternity and quality control in pathology. STR analysis showed that the primary tumor originated from the donor renal pelvis and directly extended tothe donor ureter and urinary bladder via drop metastasis.

Although the original tumor only showed focal invasion into submucosal tissue, the large metastatic foci in the left obturator lymph node and the wide spreading to the graft ureter and native bladder demonstrated the aggressive nature of the primary tumor, indicating the role of genetic factors.In addition, the patient lacked obvious predisposing factors,suggesting that this case represents de novo tumorigenesis in the transplant kidney. Although molecular analysis of the tumor showed a low VAF $\mathrm{MSH} 2$ mutation, it was not detected at protein level by MSI IHC, suggesting that MSI may play a very limited role in the tumorigenesis. In contrast, the amplification of TERT and loss of CDKN2A/CDKN2B detected in the tumor suggests that they may play an important role in this case.

\section{Conclusion}

While it is known that transplant recipients have an increased risk of urothelial carcinoma compared to the general population, the lack of the well-documented risk factors, such as older age at transplantation, BK polyomavirus infection, and prolonged post-transplantation history and dissemination of the tumor in this case shed light on the de novo tumorigenesis of the graft kidney within the host microenvironment. Amplification of TERT and loss of CDKN2A/CDKN2B detected in the tumor by next gene sequencing suggests that they may play an important role in this case. 


\section{Abbreviations}

STR

Short tandem repeat, TERT:Telomerase reverse transcriptase; CDKN:Cyclin dependent kinase inhibitor; RCC:Renal cell carcinoma; UC:Urothelial carcinoma; MSI:microsatellite instability; VAF:Iow variant allele frequency;

\section{Declarations}

Availability of data and materials

The dataset supporting the conclusions of this article is included within the article.

\section{Acknowledgements}

Not applicable.

\section{Funding}

No funding.

\section{Author information}

\section{Affiliations}

1. Department of Pathology Icahn School of Medicine, New York, NY, USA

\section{Contributions}

Joyce M. Chen,; Data Collection, Manuscript writing

William Lam; Asha Reddy, Meenakshi Mehrotra;: Performed short tandem repeat analysis and next gene sequencing

1. Kenneth Haines III; Jane Houldsworth; Qiusheng Si: Manuscript editing.

The author(s) read and approved the final manuscript.

\section{Corresponding author}

Correspondence to Qiusheng Si 


\section{Ethics declarations}

\section{Ethics approval and consent to participate}

All ethical approval and consent procedures were approved by the Medical Ethical Committee of Icahn School of Medicine at Mount Sinai. According to the institution guidelines, a single case report with deidentified patient specific information, the patient's singed privacy consent is not necessary.

\section{Consent for publication}

Not applicable.

\section{Competing interests}

The authors declare no conflict of interest/competing interests in publishing the present manuscript.

\section{References}

1. Schnuelle P, Lorenz D, Trede M, Van Der Woude FJ. Impact of renal cadaveric transplantation on survival in end-stage renal failure: evidence for reduced mortality risk compared with hemodialysis during long-term follow-up. J Am Soc Nephrol. 1998;9(11):2135.

2. Collins AJ, Foley RN, Chavers B, Gilbertson D, Herzog C, Johansen K, Kasiske B, Kutner N, Liu J, St Peter W, Guo H, Gustafson S, Heubner B, Lamb K, Li S, Li S, Peng Y, Qiu Y, Roberts T, Skeans M, Snyder J, Solid C, Thompson B, Wang C, Weinhandl E, Zaun D, Arko C, Chen SC, Daniels F, Ebben J, Frazier E, Hanzlik C, Johnson R, Sheets D, Wang X, Forrest B, Constantini E, Everson S, Eggers P, Agodoa L. United States Renal Data System 2011 Annual Data Report: Atlas of chronic kidney disease \&amp; end-stage renal disease in the United States. Am J Kidney Dis. 2012 Jan; 59(1 Suppl 1):A7, e1-420.

3. Stallone G, Infante B, Grandaliano G. Management and prevention of post-transplant malignancies in kidney transplant recipients. Clin Kidney J. 2015 Oct; 8(5):637-44.

4. Sprangers B, Nair V, Launay-Vacher V, Riella LV, Jhaveri KD. Risk factors associated with post-kidney transplant malignancies: an article from the Cancer-Kidney International Network. Clin Kidney J. 2017;11(3):315-329.

5. Kiberd BA, Rose C, Gill JS. Cancer mortality in kidney transplantation. Am J Transplant 2009; 9: 1868-1875

6. Gökçe Mi, Kocaay AF, Aktürk S, Tüzüner A. Urothelial carcinoma of the allograft kidney developed in a renal transplant patient. Turk J Urol. 2016;42(3):213-215. doi:10.5152/tud.2016.97415 
7. Michel Ortega RM, Wolff DJ, Schandl CA, Drabkin HA. Urothelial carcinoma of donor origin in a kidney transplant patient. J Immunother Cancer. 2016;4:63. Published 2016 Oct 18. doi:10.1186/s40425-016-0167-4

8. M. Roupret, A.R. Azzouzi, O. CussenotMicrosatellite instability and transitional cell carcinoma of the upper urinary tract. BJU Int, 96 (2005), pp. 489-492

9. Karami S, Yanik EL, Moore LE, Pfeiffer RM, Copeland G, Gonsalves L, Hernandez BY, Lynch CF, Pawlish K, Engels EA. Risk of Renal Cell Carcinoma Among Kidney Transplant Recipients in the United States. Am J Transplant. 2016 Dec; 16(12):3479-3489.

10. Oikawa $M$, Hatakeyama S, Fujita T. et al. BK virus-associated urothelial carcinoma of a ureter graft in a renal transplant recipient: a case report. Transplant Proc 2014; 46: 616-619

11. Yan L, Salama ME, Lanciault C. et al. Polyomavirus large T antigen is prevalent in urothelial carcinoma post-kidney transplant. Hum Pathol 2016; 48: 122-131

12. Nickeleit V, Singh HK, Goldsmith CS. et al. BK virus-associated urinary bladder carcinoma in transplant recipients: productive or nonproductive polyomavirus infections in tumor cells? Hum Pathol2013; 44: 2870-2871

13. Kausman JY, Somers GR, Francis DM. et al. Association of renal adenocarcinoma and BK virus nephropathy post transplantation. Pediatr Nephrol 2004; 19: 459-462

\section{Figures}



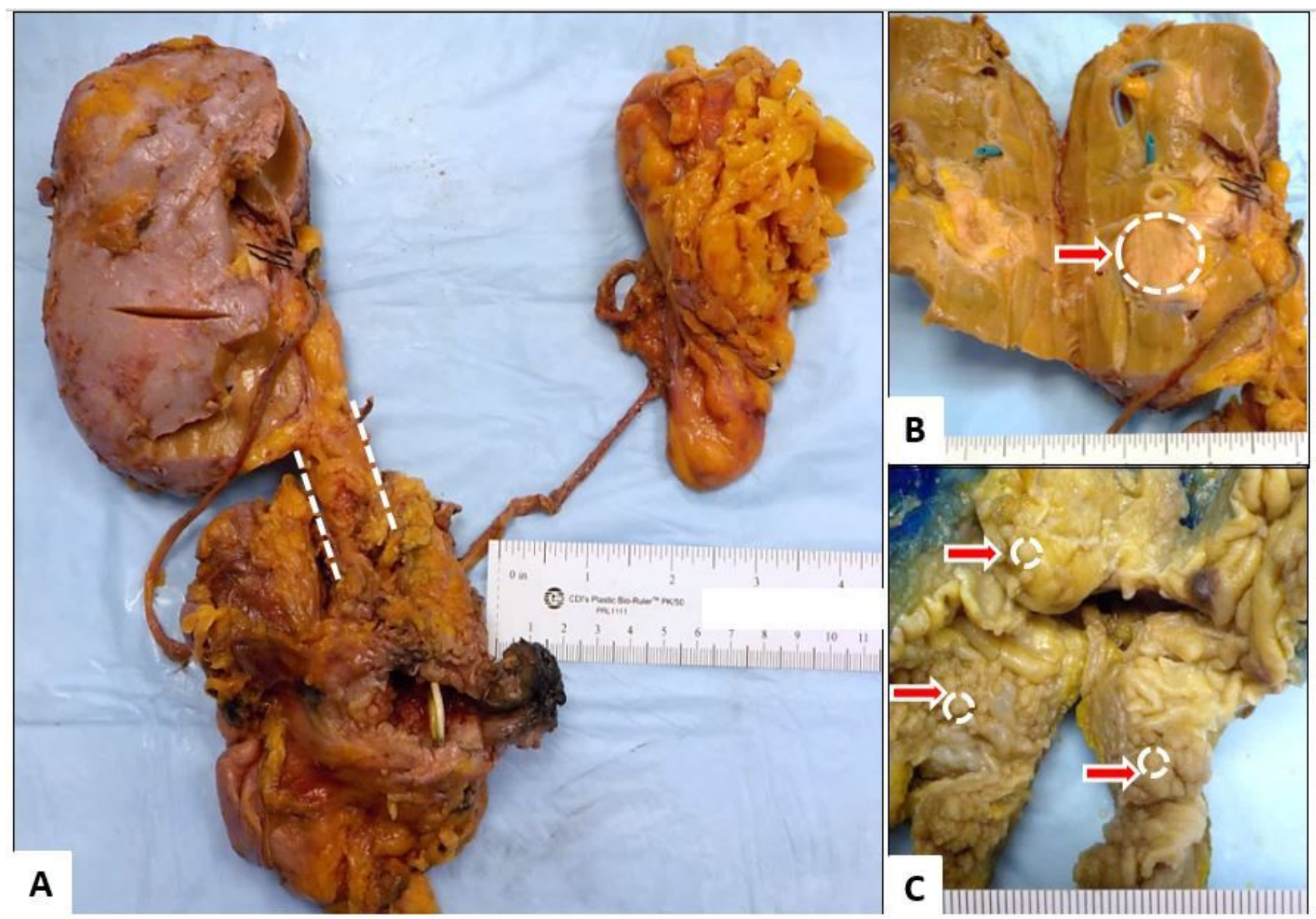

Figure 1

A, Gross photograph of the nephro-cystectomy specimen shows the bladder, donor (left) and native kidneys (right); B, Donor kidney with a renal pelvic mass; C, Native bladder with many nodules 


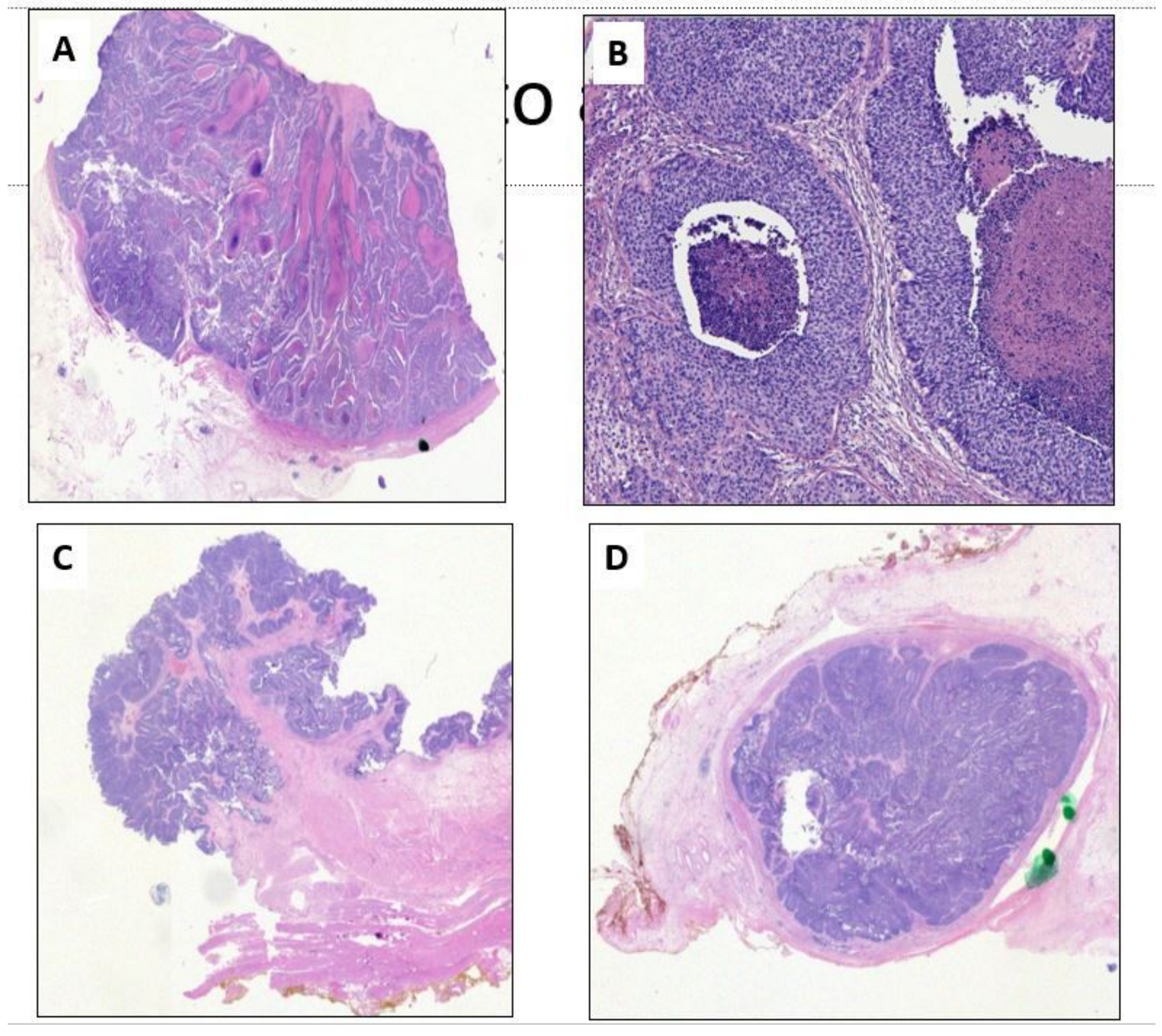

Figure 2

H\&E stain of the high-grade papillary UC of donor kidney (A\&B), urinary bladder (C) and donor ureter (D). 


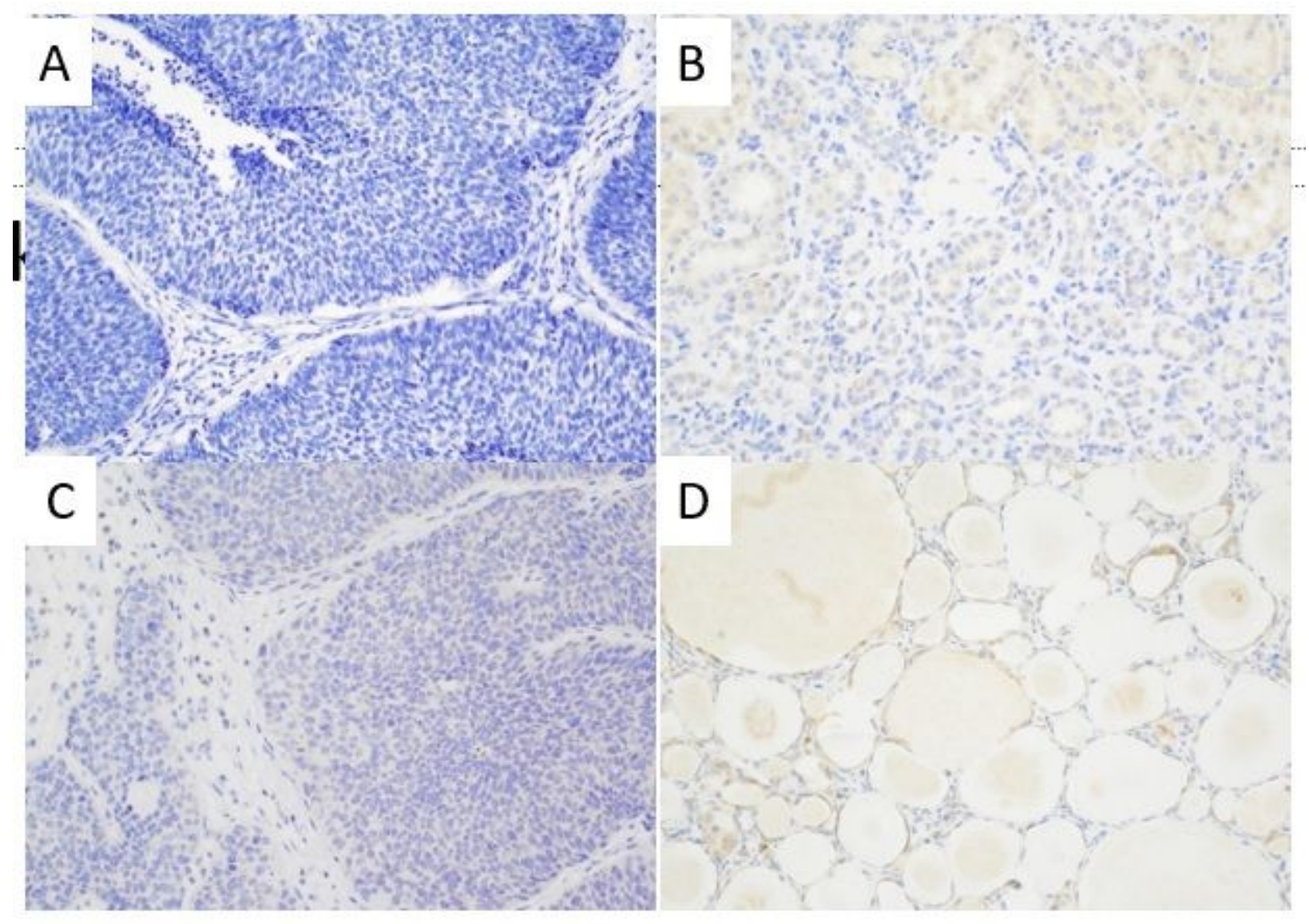

\section{Figure 3}

Negative stain of BK virus in donor-derived UC of renal pelvis (A), donor kidney (B), bladder tumor( C), and recipient kidney (D).

\section{Supplementary Files}

This is a list of supplementary files associated with this preprint. Click to download.

- CAREchecklist.pdf 